\title{
Invasive and indigenous microbiota impact intestinal stem cell activity through multiple pathways in Drosophila
}

\author{
Nicolas Buchon, ${ }^{2}$ Nichole A. Broderick, Sveta Chakrabarti, and Bruno Lemaitre ${ }^{1}$ \\ Global Health Institute, Ecole Polytechnique Federale de Lausanne (EPFL), CH-1015 Lausanne, Switzerland
}

Gut homeostasis is controlled by both immune and developmental mechanisms, and its disruption can lead to inflammatory disorders or cancerous lesions of the intestine. While the impact of bacteria on the mucosal immune system is beginning to be precisely understood, little is known about the effects of bacteria on gut epithelium renewal. Here, we addressed how both infectious and indigenous bacteria modulate stem cell activity in Drosophila. We show that the increased epithelium renewal observed upon some bacterial infections is a consequence of the oxidative burst, a major defense of the Drosophila gut. Additionally, we provide evidence that the JAK-STAT (Janus kinase-signal transducers and activators of transcription) and JNK (c-Jun $\mathrm{NH}_{2}$ terminal kinase) pathways are both required for bacteria-induced stem cell proliferation. Similarly, we demonstrate that indigenous gut microbiota activate the same, albeit reduced, program at basal levels. Altered control of gut microbiota in immune-deficient or aged flies correlates with increased epithelium renewal. Finally, we show that epithelium renewal is an essential component of Drosophila defense against oral bacterial infection. Altogether, these results indicate that gut homeostasis is achieved by a complex interregulation of the immune response, gut microbiota, and stem cell activity.

[Keywords: Intestinal stem cell; proliferation; gut microbiota; JAK-STAT; JNK; pathogenic bacteria]

Supplemental material is available at http://www.genesdev.org.

Received June 2, 2009; revised version accepted August 25, 2009.

The gut of all organisms is in constant exposure to environmental and microbial aggressions (Sansonetti 2004). It is an active barrier, limiting invasion by pathogenic microorganisms and promoting tolerance to indigenous microbiota (Pedron and Sansonetti 2008). To maintain homeostasis, the gut epithelium is constantly renewed throughout an organism's life by the division and differentiation of intestinal stem cells (ISCs). In Drosophila, ISCs are scattered along the basement membrane of the midgut (Micchelli and Perrimon 2006; Ohlstein and Spradling 2006). The division of an ISC generates both a new ISC and a post-mitotic enteroblast, which differentiates into either an absorptive enterocyte or a secretory enteroendocrine cell. In basal conditions, the adult gut epithelium is renewed in $\sim 1-2$ wk (Micchelli and Perrimon 2006).

We recently reported a global analysis of the changes in gene expression that occur in the Drosophila gut in response to infection by a Gram-negative bacterium, Erwinia carotovora carotovora 15 (Ecc15) (Buchon et al. 2009). This study demonstrated that bacterial infec-

Corresponding authors.

${ }^{1}$ E-MAIL bruno.lemaitre@epfl.ch; FAX 41-21-693-17-90.

${ }^{2}$ E-MAIL nicolas.buchon@epfl.ch; FAX 41-21-693-17-90.

Article is online at http://www.genesdev.org/cgi/doi/10.1101/gad.1827009. tion triggers expression of a combination of immune, stress, and developmental signaling pathways. Most immune genes-notably, antibacterial peptide genes (e.g., Diptericin)—are induced in the gut by the Imd pathway. This pathway is activated upon recognition of peptidoglycan of Gram-negative bacteria by the transmembrane receptor PGRP-LC, and leads to activation of the NF- $\mathrm{KB}$ protein Relish (Zaidman-Remy et al. 2006). Subsequent analysis showed that the JAK-STAT (Janus kinase-signal transducers and activators of transcription) signaling pathway was activated by damage in the gut of infected flies and regulates the production of a subset of antimicrobial peptides. Surprisingly, it was further observed that ingestion of Ecc15 strongly stimulates ISC division, promoting a rapid turnover of the gut epithelium (Buchon et al. 2009). These results highlighted an unexpected link between oral bacterial infection and epithelial renewal. These data also suggested that, following infection, gut homeostasis is maintained by the balance between cell damage induced by infection and epithelial repair by ISC activation. However, the precise causes of ISC proliferation, as well as the signaling events regulating this induction, were unclear, as was the effect of indigenous or lethal bacteria on this mechanism.

Here we use the genetically tractable model Drosophila melanogaster to study the link between gut bacteria, the 
gut immune response, and epithelium renewal. We show that the oxidative burst, induced by the host consecutive to Ecc15 infection, is a major inducer of ISC activation. Infection with this bacterium activates both JAK-STAT and JNK (c-Jun $\mathrm{NH}_{2}$ terminal kinase) pathways in ISCs to promote proliferation. Indigenous bacteria induce the same program and lead to a basal level of proliferation. Inversely, Pseudomonas entomophila, a lethal entomopathogenic bacterium, interferes with ISC activation, thereby disrupting gut homeostasis.

\section{Results}

Infection-induced oxidative burst is a major inducer of epithelium renewal

The gut immune response of Drosophila has two main components: an initial oxidative burst by the NADPH oxidase enzyme Duox (Ha et al. 2005a), followed by the synthesis of antimicrobial peptides controlled by the Imd pathway. Previously, we reported that oral infection with Ecc15 stimulates epithelium turnover in the intestine and strongly activates the transcription of genes known to respond to oxidative stress (Buchon et al. 2009). Since Ecc15 is known to induce Duox activity (Ha et al. 2005a), we hypothesized that the oxidative burst induced upon ingestion of Ecc15 could be a major inducer of both gut damage and epithelium renewal. To test this, we monitored epithelium renewal following ingestion of Ecc15 in flies in which the level of reactive oxygen species (ROS) had been artificially reduced. Intestinal epithelium renewal can be monitored easily in Drosophila by (1) counting the number of dividing cells along the midgut using an anti-phosphohistone $\mathrm{H} 3$ (anti-PH3) antibody as an indicator of mitotic activity; (2) using the esgGal4, $U A S$-GFP marker, which strongly labels ISCs, enteroblasts, and newly synthesized enterocytes; or (3) by cell lineage analysis revealing the expansion of lacZ-marked clones; with the two latter monitoring not only cell division, but also epithelium differentiation (Micchelli and Perrimon 2006; Ohlstein and Spradling 2006). As shown previously, we detected a massive epithelium renewal following ingestion of Ecc15 by both clonal analysis and the stem cell marker esgGal4, UAS-GFP. In contrast, epithelium renewal did not increase in flies fed Ecc15 in combination with the antioxidants $\mathrm{N}$-acetyl cysteine (NAC) or glutathione (Fig. 1A,B; quantification in Supplemental Fig. S1C), although these two compounds did not affect Ecc15 viability (Supplemental Fig. S1A,B). Conversely, ingestion of paraquat, a potent inducer of ROS, induced epithelium renewal without infection (Fig. 1A,B), in agreement with previous studies (Biteau et al. 2008; Buchon et al. 2009; Chatterjee and Ip 2009).

While increased ISC proliferation was observed in Imddeficient flies infected orally with Ecc15 (Buchon et al. 2009), silencing of the Duox gene by RNAi significantly reduced the number of mitotic ISCs detected with antiPH3 (Fig. 1C). Similarly, ISC proliferation was also reduced in flies carrying either a mutation in $G \alpha q$, which
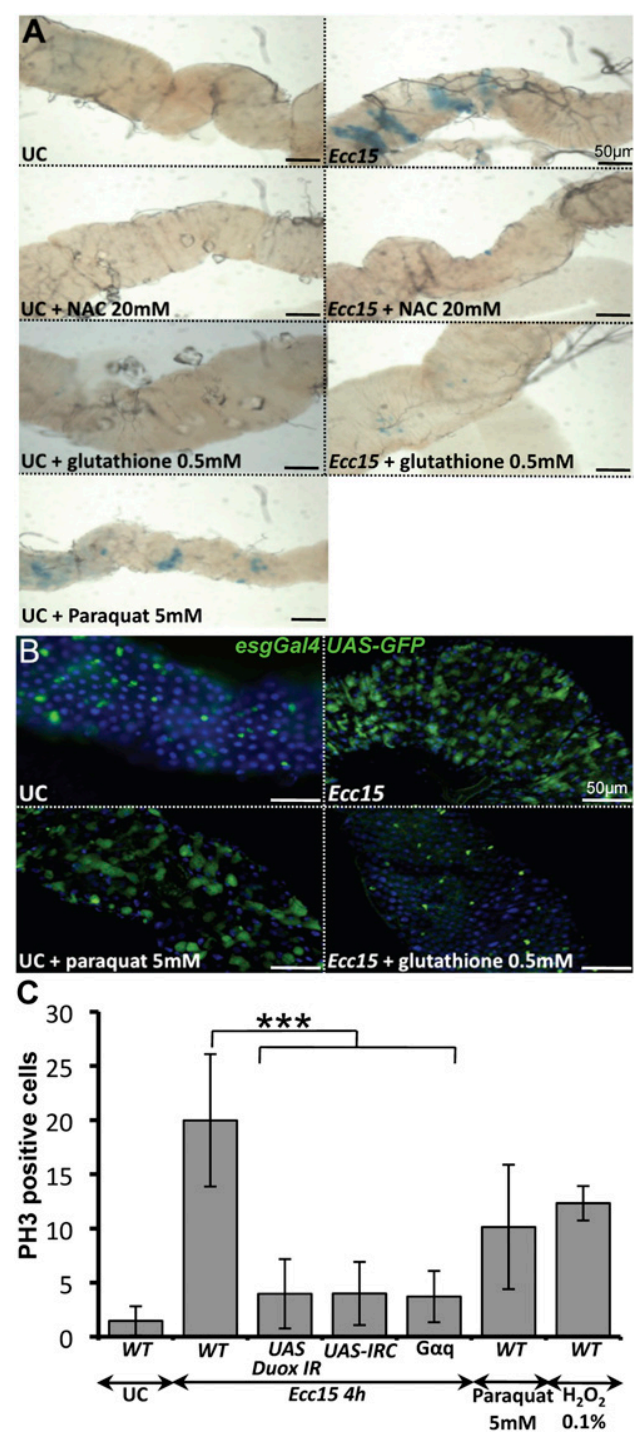

Figure 1. Immune-stimulated oxidative burst induces stem cell activation in the gut. (A) Large lacZ-marked clones containing the tubulin promoter-lacZ fusion due to mitotic recombination are observed in the guts of flies orally infected with Ecc15 or fed with paraquat. No expansion of lacZ clones was detected in the gut of flies fed with both Ecc15 and antioxidants (NAC or gluthathione). Quantification of lacZ clones is shown in Supplemental Figure S1C. (B) Ingestion of Ecc15 or paraquat induces a marked increase in the number of esgGal4, UAS-GFPpositive cells. In contrast, coingestion of Ecc15 and glutathione did not induce epithelium renewal. In unchallenged flies, most of the GFP signal corresponds to ISCs. In Ecc15-infected flies, the GFP signal was observed in both ISCs and ISC-derived daughter cells (see Supplemental Fig. S6 for distribution of escargot-positive cells along the midgut). Flies were collected $16 \mathrm{~h}$ post-infection. $(C)$ Quantification of $\mathrm{PH} 3$-positive cells per midgut of unchallenged (UC) flies or flies collected $4 \mathrm{~h}$ after feeding with Ecc15, paraquat, or $\mathrm{H}_{2} \mathrm{O}_{2}$. Guts of Duox RNAi flies (daGal4, UAS-Duox-IR), Gaq-deficient flies, or flies overexpressing a catalase (daGal4, UAS-IRC) orally infected with Ecc15 exhibit a lower number of dividing ISCs compared with wild type. Mean \pm standard deviation (SD) are shown. Values significantly different in a Student's $t$-test $(P<0.001)$ are denoted by three asterisks $\left({ }^{\star \star \star}\right)$. 
encodes a heterodimeric $\mathrm{G}$ protein responsible for Duox enzymatic activation (Ha et al. 2009), or overexpressing IRC, a catalase involved in ROS detoxification (Fig. 1C; $\mathrm{Ha}$ et al. 2005b). Collectively, our results indicate that the increased epithelium renewal observed upon infection with Ecc15 is largely a consequence of the oxidative burst resulting from Duox activation in response to infection. Reduced ISC proliferation in flies with reduced Duox activity or coinfected with Ecc15 and antioxidants was correlated with reduced levels of cell death in the gut (data not shown). This suggests that the increase in epithelium renewal is not a direct effect of ROS, but rather an effect of the damage caused by the oxidative burst. In agreement with this, the ingestion of toxic compounds such as SDS, DSS, or bleomycin is sufficient to induce epithelium renewal (Amcheslavsky et al. 2009; Buchon et al. 2009).

Activation of the JAK-STAT pathway in ISCs by the cytokine Upd3 stimulates epithelium renewal upon Ecc15 infection

We next investigated the nature of the signaling pathways stimulating epithelium renewal upon Ecc15 infection. The JAK-STAT pathway has been implicated in stem cell control in Malpighian tubules, testis, and ovaries (Gregory et al. 2008). We demonstrated previously that three genes encoding components of the JAK-STAT pathway - the receptor Domeless; one of its ligands, Unpaired $3(\mathrm{Upd} 3)$; and a repressor of the receptor/JAK complex, Socs36E-were strongly induced in the gut following ingestion of Ecc15 and paraquat (Buchon et al. 2009). Interestingly, the level of JAK-STAT signaling activity in Ecc15-infected guts was reduced in Duox RNAi flies or flies fed with antioxidants, as revealed by the decreased induction of the target genes upd 3 and Socs36E, and of the JAK-STAT reporter gene STAT-GFP (Fig. 2A; Bach et al. 2007; data not shown). Moreover, use of the STAT-GFP reporter gene revealed that the JAK-STAT pathway is already active at a basal level in ISCs and enteroblasts of unchallenged flies (Supplemental Fig. S2A). The strong correlation between stimuli activating stem cell proliferation and those inducing JAK-STAT activity prompted us to examine the role of the JAK-STAT pathway in controlling ISC proliferation upon bacterial infection.

In this aim, we expressed RNAi or dominant-negative constructs targeting JAK-STAT pathway components in either ISCs (using esgGal4) or enterocytes (using NP1Gal4) and monitored stem cell activity. Stem cell proliferation in the midgut, as monitored by the number of PH3-positive cells, was still observed in flies with reduced STAT activity in enterocytes (NP1Gal4,UASSTAT92E-IR) (Fig. 2B). The mitotic index was even higher than in wild-type flies. Since the expression of a STAT92E-IR construct driven by esgGal4 resulted in lethality, we generated conditional STAT92E-IR individuals by combining the esgGal4 transgene and a thermosensitive version of the Gal4 inhibitor, tubGal80 ${ }^{T S}$ (referred to as esgGald ${ }^{T S}$ ). When reared at a restrictive temperature $\left(18^{\circ} \mathrm{C}, \mathrm{Gal} 80^{\mathrm{TS}}\right.$ on, Gal4 system off) during their entire development, adult flies displayed a wild-type number and distribution of ISCs. However, shifting flies to a permissive temperature $\left(29^{\circ} \mathrm{C}, \mathrm{Gal} 80^{\mathrm{TS}}\right.$ off, Gal4 system on) for $3 \mathrm{~d}$ to deplete STAT in ISCs changed the pattern of escargot-positive cells along the midgut (Fig. 2C; Supplemental Fig. S2B). In the anterior midgut, we observed a reduction in the number of escargot GFPpositive cells. However, small aggregates of escargot GFPpositive cells were observed occasionally in the anterior midgut of esgGal4 ${ }^{T S}$ STAT92E-IR flies (indicated by an arrow in Fig. 2C3). In contrast, a high number of GFPpositive cells were consistently observed in the posterior region of the midgut. These cells were small-nucleated and did not express the ISC marker Delta or the enteroendocrine marker Prospero, raising the possibility that they are enteroblasts (Supplemental Fig. S2C,D). This suggests that the JAK-STAT pathway is not required for ISC proliferation, but rather for the proper differentiation of ISC progeny in basal turnover conditions. In contrast to wild-type flies, no increase in the number of mitotic cells (PH3positive) nor change in the pattern and distribution of escargot GFP-positive cells was observed in the gut of esgGal4 ${ }^{T S}$ STAT92E-IR flies upon Ecc15 infection (Fig. $2 B, C$ ), indicating a lack of epithelium renewal induction. Similar results were obtained with the use of the dominant-negative version of the JAK-STAT pathway receptor Domeless (Fig. 2B; Brown et al. 2001; data not shown).

We further tested the effect of reducing JAK-STAT signaling by generating mosaic animals with clones that are mutant for the STAT92E transcription factor (Lee and Luo 2001; Krezmien et al. 2007). Upon Ecc15 infection of the mosaic flies, STAT92E mutant clones were rarely observed, but when present were composed of only smallnucleated cells. In contrast, control clones contained both small diploid progenitors and large polyploid enterocytes (Supplemental Fig. S2E). This indicates that a reduction in JAK-STAT signaling in ISCs blocks epithelium renewal induced upon Ecc15 infection. Finally, overexpressing a gain-of-function form of the JAK kinase Hop in ISCs (UAS-hop ${ }^{\text {Tum }}$ ) triggered epithelium renewal without infection (Fig. 2B,D1). We conclude that the JAK-STAT pathway is required in ISCs for the increased epithelium renewal induced upon bacterial infection with Ecc15.

We reported previously that the JAK-STAT ligand upd3 is transcriptionally induced in the gut upon ingestion of both Ecc15 and paraquat (Buchon et al. 2009). Use of an upd3-Gal4; UAS-GFP reporter transgene (Agaisse et al. 2003) revealed that upd3 was expressed mostly in enterocytes (Fig. 2E). In agreement, upd3 expression was decreased in flies when upd3-IR was expressed in enterocytes, but not when it was expressed in ISCs (Supplemental Fig. S2F). Interestingly, physical injury of the gut with forceps rapidly induced a localized expression of upd3 (Fig. 2F), demonstrating that $\mathrm{Upd} 3$ is induced in the vicinity of damaged enterocytes and supporting its role in the activation of the JAK-STAT pathway in ISCs. Accordingly, specific silencing of upd3 expression in enterocytes, but not in ISCs, strongly decreased the number of proliferative ISCs in response to infection (Fig. 2B). Conversely, the ectopic expression of upd3 in ISCs was 

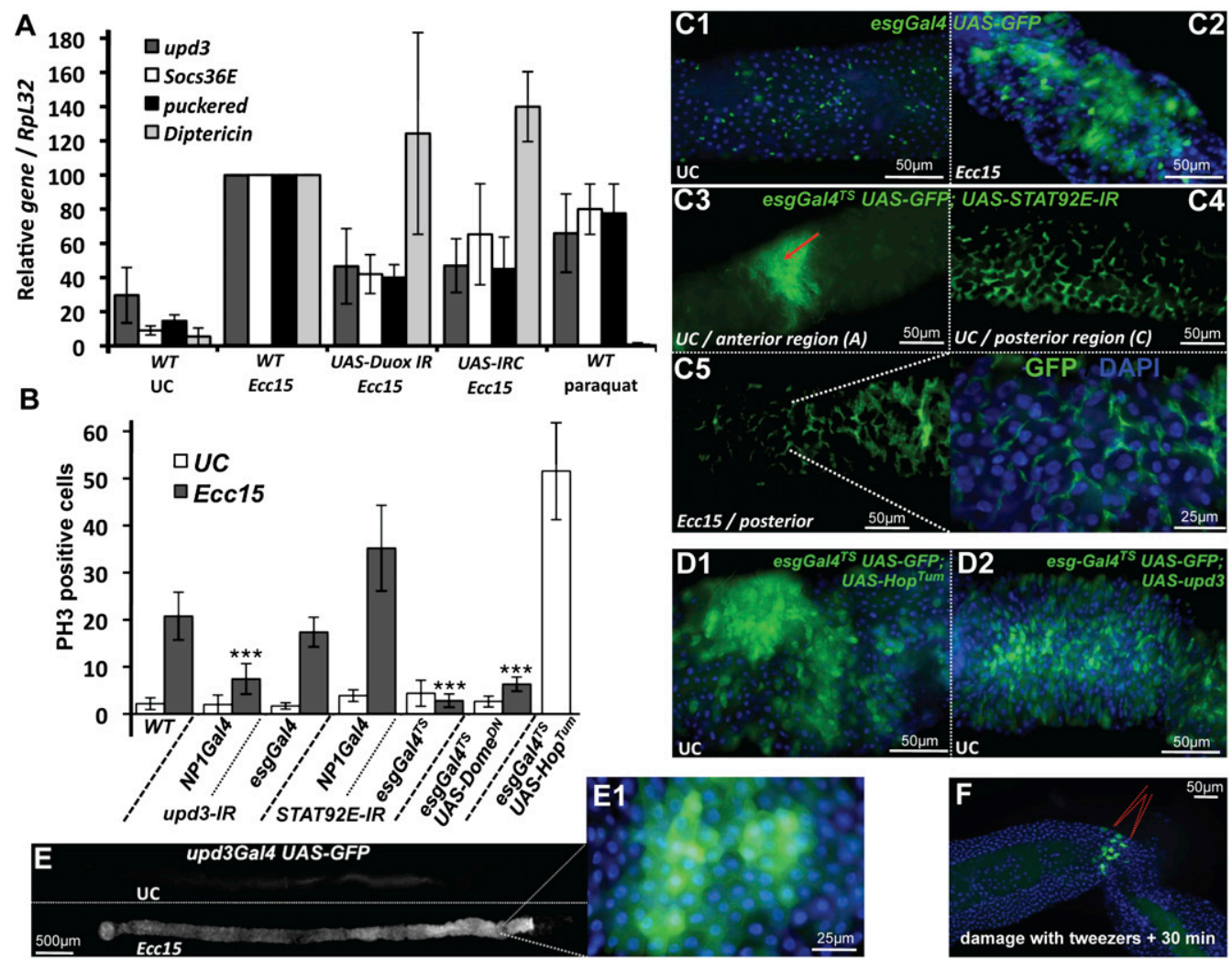

Figure 2. The JAK-STAT pathway is required for ISC proliferation upon Ecc15 infection. (A) Oxidative stress modulates the activation of JAK-STAT and JNK pathways upon Ecc15 infection. RT-qPCR analysis of gut extracts shows that the expression levels of JAK-STAT (upd3, Socs36E, monitored $4 \mathrm{~h}$ post-infection) and JNK (puckered, monitored 30 min post-infection) target genes were reduced upon Ecc15 infection in the gut of Duox RNAi flies or flies expressing a catalase (daGal4; UAS-IRC). Ingestion of paraquat was sufficient to induce upd3, Socs36E, or puckered. In contrast, the level of Diptericin (a gene tightly regulated by the Imd pathway) was not affected by conditions modulating ROS. Mean of four independent experiments \pm SD are shown. (B) Quantification of PH3-positive cells per midgut shows an increase in the number of mitotic cells upon Ecc15 infection in both wild-type flies and flies with reduced JAK-STAT activity in enterocytes (NP1Gal4, UAS-STAT92E-IR), but not in ISCs (esgGal4 ${ }^{T S}$, UAS-STAT92E-IR). Silencing of upd3 in enterocytes (NP1Gal4, UAS-upd3-IR), but not in ISCs (esgGal4, UAS-upd3-IR), reduced the number of mitotic ISCs in the guts of flies collected $4 \mathrm{~h}$ after feeding with Ecc15. Overexpression of a gain-of-function allele of hop in ISCs increases the mitotic index lesgGal4 ${ }^{T S}$, UAShop $\left.{ }^{\text {Tum }}\right)$. Mean \pm SD are shown. Values significantly different from infected wild-type flies in a $t$-test at $P<0.001$ are denoted by three asterisks $\left(^{\star \star \star}\right) .(C 1-C 5)$ A loss of stem cells was observed in the anterior region of esgGa14 ${ }^{T S}, U A S-S T A T 92 E-I R$ flies raised at $29^{\circ} \mathrm{C}$. In some flies, accumulation of small-nucleated escargot-positive cells were observed in the anterior part of the midgut, as indicated with a red arrow in C3. (C4) Accumulation of small-nucleated escargot-positive cells was observed in the posterior regions of the gut of all esgGa14 ${ }^{T S}$, UAS-STAT92E-IR flies raised at $29^{\circ} \mathrm{C}$, in the absence of infection. (C5) No increase in epithelium renewal was observed in these flies upon infection with Ecc15, as indicated by the lack of large nuclei cells expressing GFP. Expression of the esgGal4, UAS-GFP reporter gene was monitored $16 \mathrm{~h}$ after infection with Ecc15. $(D 1, D 2)$ Overexpression of hop Tum (D1) or upd3 (D2) in ISCs is sufficient to induce a high level of epithelium renewal in the absence of infection. (E) upd3 expression is induced along the gut following Ecc15 ingestion. (E1) upd3 was expressed mostly in enterocytes $4 \mathrm{~h}$ after Ecc15 infection, as revealed by the GFP signal (green) of upd3Gal4 UAS-GFP flies. $(F)$ Physical damage to the gut with tweezers induced local expression of the upd3Gal4, UAS-GFP reporter within 30 min following injury.

sufficient to induce a high level of epithelium renewal in the absence of infection (Fig. 2D2). Altogether, these results indicate that upon Ecc15 infection, enterocytes release Upd3, which activates the JAK-STAT pathway in ISCs to promote proliferation and to repair damage to the gut.

\section{The INK pathway is required for ISC maintenance} and proliferation in the gut of infected flies

The JNK pathway is activated by a variety of environmental challenges, including oxidative stress, and was demonstrated recently to be required in ISCs to maintain gut homeostasis in aging flies (Wang et al. 2003; Biteau et al. 2008). We reported previously that the JNK pathway is activated in the gut of flies soon after ingestion of Ecc15 (Buchon et al. 2009). Furthermore, coimmunostaining of the ISC marker esg-GFP and of puckered (puc-lacZ), a reporter of JNK pathway activity (Martín-Blanco et al. 1998), revealed that the JNK pathway was strongly activated in both enterocytes and ISCs upon Ecc15 infection (Fig. 3A). Interestingly, RT-qPCR analysis revealed that JNK induction was dampened in both Duox 

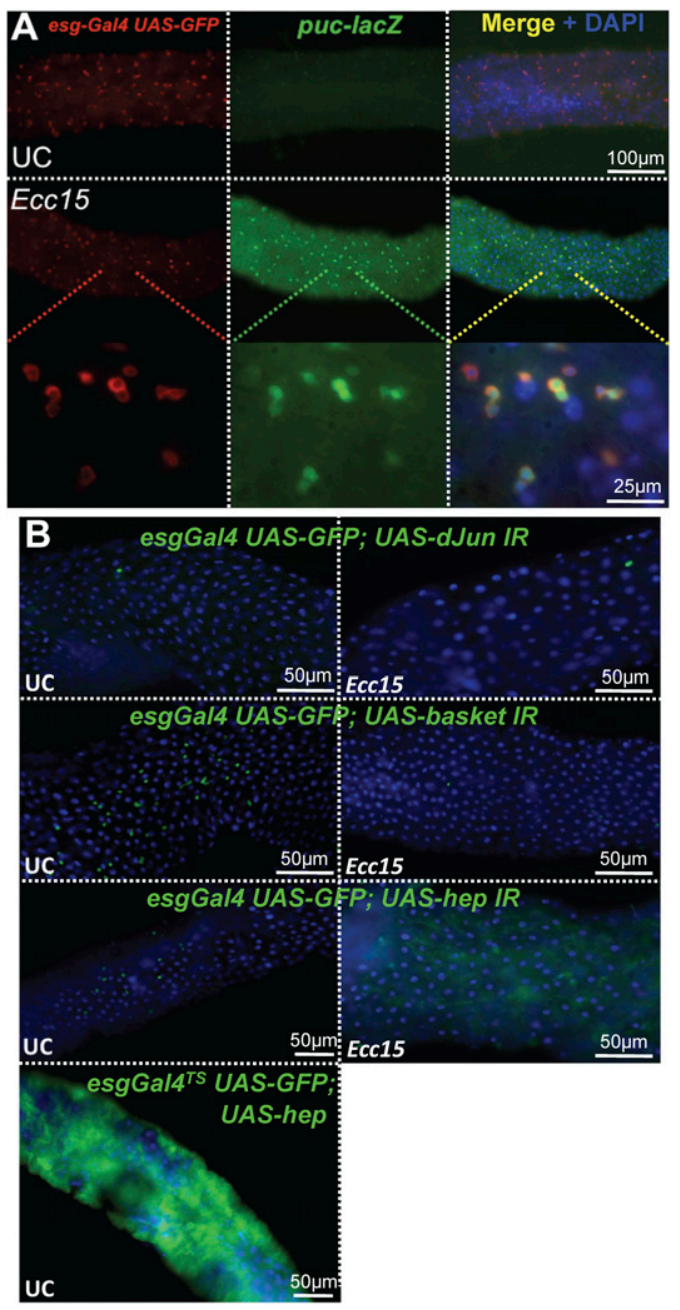

Figure 3. The JNK pathway is required for ISC maintenance and proliferation upon Ecc15 infection. (A) Immunostaining with antibodies against GFP (red) or LacZ (green) of esgGal4, UAS-GFP, puc ${ }^{E 69}$ (puc-lacZ) flies collected $30 \mathrm{~min}$ after Ecc15 infection shows that the level of JNK signaling activity increases (top center panel, compared with middle center panel) upon Ecc15 infection in both enterocytes and ISCs, the staining being more intense in ISCs (merge, right panels); higher magnification is shown in the bottom panels. $(B)$ Loss of ISCs and the absence of epithelium renewal were observed in the guts of flies with reduced JNK activity in ISCs. Guts of flies carrying the esgGal4, UAS-GFP combined with UAS-dJun-IR, UAS-basket $I R$, or UAS-hep-IR were examined $16 \mathrm{~h}$ post-infection with Ecc15. Silencing of $d J u n-I R$ in ISCs reduced the number of ISC in both unchallenged and challenged animals. In contrast, silencing of the genes encoding the JNK Basket and JNKK Hep in ISCs leads to a loss of ISCs only after infection with Ecc15. Ectopic expression of UAS-hep in ISCs induces epithelium renewal in the absence of infection.

RNAi flies and flies coinfected with Ecc15 and antioxidants, as monitored by a decrease in expression of puckered, a target gene of the JNK pathway (Fig. 2A). These results prompted us to investigate the involvement of the JNK pathway in ISC proliferation during bacterial infection. Silencing the transcription factor d/un in ISCs by RNAi resulted in the loss of ISCs along the gut of unchallenged flies, as indicated by the absence of escargot GFP-positive cells (Fig. 3B). Immunostaining with the ISC-specific marker Delta confirmed that the absence of esgargot GFP-positive cells corresponded with the loss of ISCs (data not shown). Similar results were obtained when the inducible esg-Gal4 tubGAL80 ${ }^{\text {ts }}$ system was used to drive the dIun-IR construct, indicating that the loss of ISCs does not result from an effect of JNK during development (data not shown). Interestingly, knockdown of the JNK basket or the JNK kinase (JNKK) hemipterous (hep) in ISCs or overexpression of a negative regulator of JNK signaling (UAS-puckered) did not alter the number of ISCs in unchallenged flies (Fig. 3B; data not shown). However, upon Ecc15 infection, the number of ISCs was decreased dramatically in the gut of flies with reduced JNK activity, and no epithelium renewal was observed (Fig. 3B). Moreover, immunostaining for the apoptotic marker caspase3 revealed that, upon Ecc15 infection, some remaining ISCs were undergoing apoptosis when depleted for the JNK Basket (Supplemental Fig. S3A). Importantly, no increase in the number of dividing cells was observed upon Ecc15 infection in the gut of flies with reduced JNK activity in ISCs, as monitored by the number of PH3-positive cells (Supplemental Fig. S3B). In contrast, overexpression of hep with the esg-Gal4 ${ }^{T S}$ driver led to increased epithelium renewal in the absence of infection (Fig. 3B; Supplemental Fig. S3B).

We conclude that the lack of JNK pathway activity in ISCs results in a premature loss of ISCs and the consequent absence of epithelium renewal upon bacterial infection with Ecc15.

\section{Epithelium renewal is an essential component of Drosophila defense to Ecc15}

Both wild-type and Imd-deficient flies survive ingestion of Ecc15 (Basset et al. 2000), indicating that even without a functional Imd-dependent immune response, this bacterium is not lethal. Importantly, we observed no difference in the level of epithelium renewal between wildtype and Imd-deficient flies in response to Ecc15 (Buchon et al. 2009). In contrast, reduction of JNK or JAK-STAT signaling in ISCs abolishes the epithelium renewal that is induced upon infection with Ecc15, but does not affect the Imd-dependent immune response (data not shown). To analyze the extent to which epithelium renewal is important in the resistance to Ecc15 infection, we compared the survival of flies in which the activity of the JNK (UAS-basket-IR, UAS-dJun-IR), JAK-STAT (UAS-upd3IR or UAS-STAT92E-IR), and Imd (UAS-dFadd-IR) pathways had been selectively reduced in either enterocytes (Fig. 4A) or ISCs (Fig. 4B). Strikingly, flies impaired in their capacity to renew their epithelium-due to the inhibition of either JAK-STAT or JNK signaling in ISCs, or the silencing of upd3 in enterocytes-succumbed 4-8 $\mathrm{d}$ following ingestion of Ecc15 (Fig. 4A,B). Examination of flies impaired in epithelium renewal at $4 \mathrm{~d}$ revealed that their guts were morphologically altered, with regions devoid of enterocytes (Fig. 4C) or displaying aberrant cell 

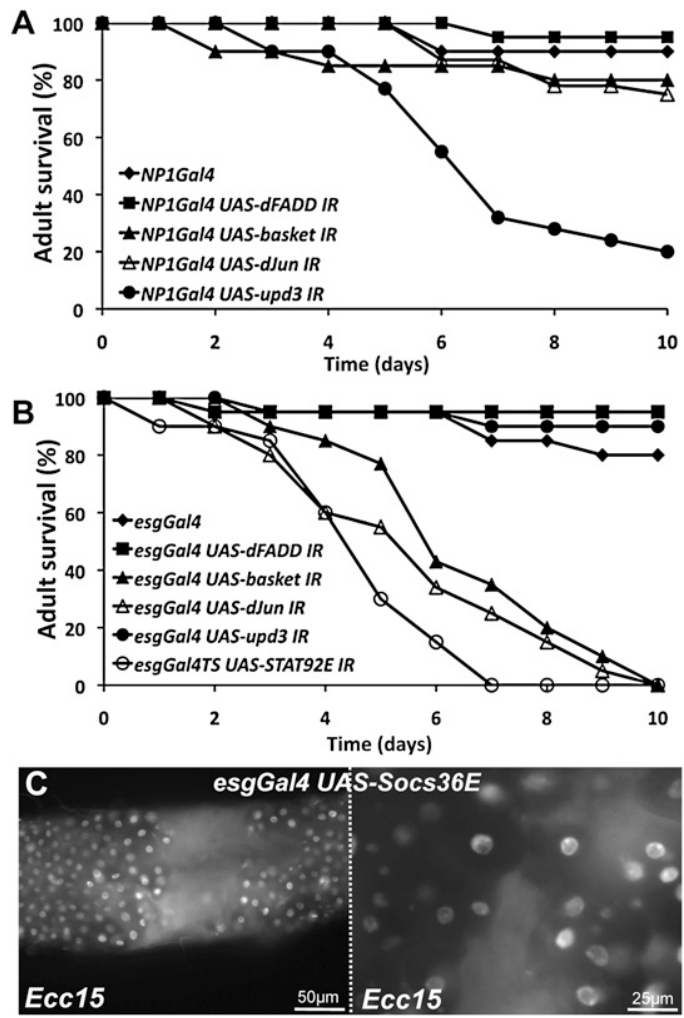

Figure 4. Epithelium renewal is required for proper resistance to Ecc15 infection. $(A, B)$ Survival analysis at $29^{\circ} \mathrm{C}$ shows that flies impaired in epithelium renewal succumbed 4-8 d following ingestion of Ecc15. UAS-dFADD-IR, UAS-basket-IR, UASdJun-IR, or UAS-upd3-IR constructs were expressed in either enterocytes (NP1Gal4; A) or ISCs (esgGal4 or esgGal4 $\left.{ }^{T S} ; B\right) .(C)$ Guts of flies impaired in epithelium renewal displayed altered gut morphology as revealed by the lack of DAPI nuclear staining. Flies were observed $4 \mathrm{~d}$ post-infection.

shapes, suggesting a disruption in the continuity of the gut and a lack of tissue integrity. The corresponding increase in susceptibility demonstrates that epithelium renewal is necessary for the proper recovery of flies after infection with Ecc15 and is more critical than the Imd pathway in the response to this bacterium.

\section{Indigenous microbiota impact epithelium renewal through ISC stimulation}

Even in basal conditions, the gut is permanently in contact with indigenous microorganisms (Hooper 2004). We used axenically raised flies to investigate how gut microbiota impact epithelium renewal. Both the number of mitotic ISCs and the rate of epithelium renewal were reduced in axenic flies as compared with conventionally raised (CR) flies (Fig. 5A; Supplemental Fig. S4A,B). This reduction in epithelium renewal was not due to an inability of axenic flies to activate renewal, as infection with Ecc15 and reintroduction of culturable indigenous bacteria increased mitotic activity in these flies (data not shown). Moreover, RT-qPCR analysis demonstrated that the gut microbiota regulate, to some extent, the basal levels of JAK-STAT, JNK, and Imd pathways (Fig. 5B). Use of the upd3Gal4, UAS-GFP reporter gene revealed that upd 3 was expressed at low levels in a few cells scattered along the gut of CR flies. In sharp contrast, no expression of upd3 was observed in the gut of axenic flies (Fig. 5C). Taken together, these data suggest that, under normal conditions, gut microbiota stimulate these pathways, promoting a basal level of epithelium renewal. Interestingly, higher numbers of dividing cells were detected in the absence of infection in the guts of Relish ${ }^{E 20}$ or PGRP$L C^{E 12}$ flies, which are deficient for the Imd pathway (Fig. 5A). These flies harbor 10-fold higher counts of indigenous bacteria than wild-type (Supplemental Fig. S4C), suggesting that an abnormally abundant microbiota could increase epithelium renewal. To test this hypothesis, we raised Relish ${ }^{E 2 O}$ and PGRP-LC ${ }^{E 12}$ flies in axenic conditions and monitored ISC proliferation by anti-PH3 staining. Increased ISC proliferation was no longer observed in Relish ${ }^{E 2 O}$ and PGRP-LC $C^{E 12}$ axenic flies (Fig. 5A). These data indicate that indigenous bacteria condition the basal level of epithelium renewal by stimulating ISC division, probably through an increase of JAK-STAT and JNK activity.

Recent studies have revealed that age-related deterioration of the intestinal epithelium is associated with excessive ISC proliferation and aberrant differentiation (Biteau et al. 2008; YJ Choi et al. 2008; Park et al. 2009). In agreement with this, we observed a 10 -fold higher mitotic index in the midguts of 30-d-old flies as compared with 3-d-old flies by anti-PH3 staining (Fig. 5A). Moreover, increased esgGal4, UAS-GFP signal was observed in the guts of 30-d-old flies (Fig. 5D1), at a similar level to that observed with Ecc15 infection. Higher magnification revealed the accumulation of large, rounded escargotpositive cells, described previously as a hallmark of gut disorganization upon aging (Fig. 5D2; Biteau et al. 2008). This deregulation of epithelium renewal correlated with increased expression levels of target genes of the JNK, JAK-STAT, and Imd pathways (Fig. 5B). These agedependant symptoms were exacerbated in the Relish ${ }^{E 2 O}$ flies (Fig. 5A), whose guts were morphologically altered with regions devoid of enterocytes (Supplemental Fig. S4D). Interestingly, guts from both wild-type and Relish $^{E 2 O}$ 30-d-old flies contain higher counts of indigenous bacteria than their younger counterparts (Supplemental Fig. S4C; Ren et al. 2007), raising the possibility that the increased epithelium renewal in older flies could be caused by the abundant microbiota. We examined axenically raised flies to investigate how gut microbiota impact age degeneration of the gut. Strikingly, guts from $30-$ d-old flies raised axenically have levels of epithelium renewal more similar to that of young flies, as indicated by the mitotic index and use of the esgGal4, UAS-GFP marker (Fig. 5A,D). This correlates with reduced levels of JNK and JAK-STAT activity (Fig. 5B). Moreover, the guts of old Relish ${ }^{E 2 O}$ axenic flies do not exhibit alterations in intestinal integrity (Supplemental Fig. S4D). These data indicate that age-related defects in the gut are largely reverted in the absence of indigenous bacteria. 

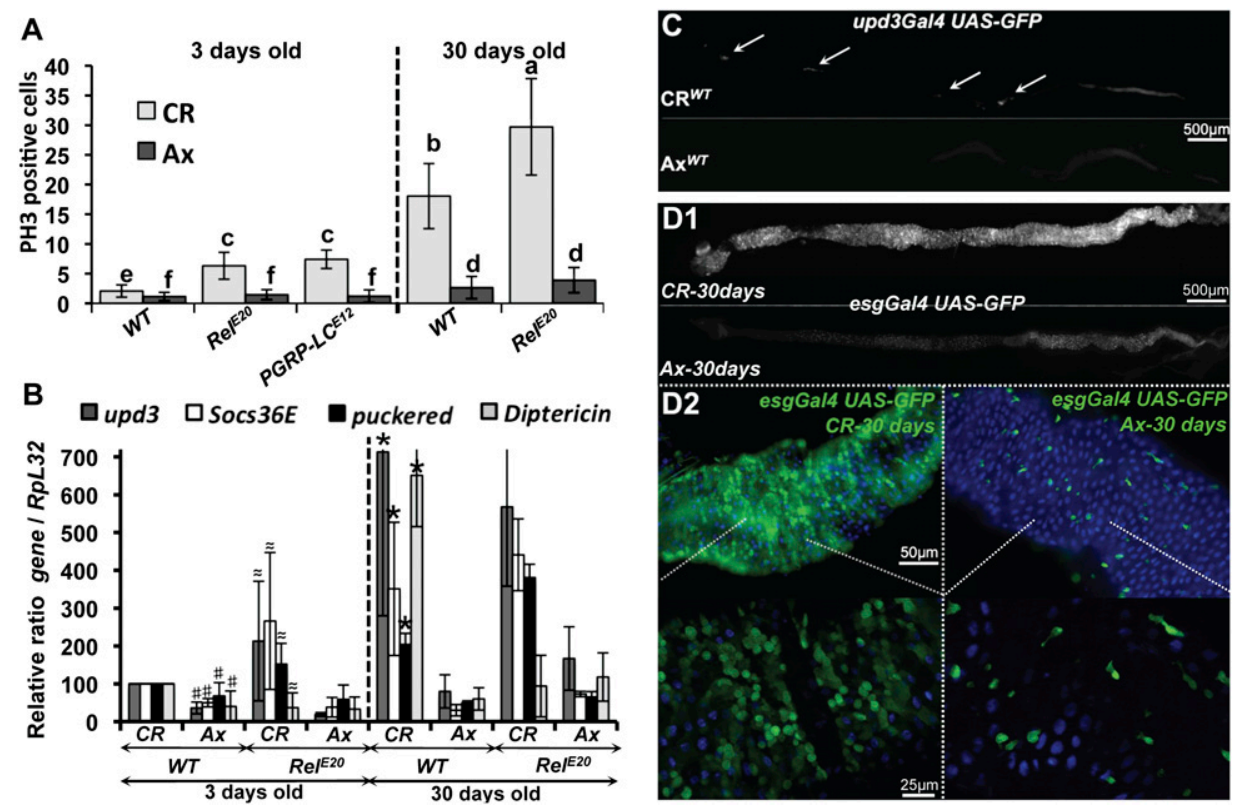

Figure 5. Indigenous gut microbiota activate a basal level of ISC activity. (A) Axenic (Ax) flies display a level of ISC proliferation lower than CR flies, as measured by the number of mitotic cells along the gut of unchallenged flies (PH3-positive cells). Higher numbers of dividing cells were detected in the guts of CR flies, but not axenic Rel ${ }^{E 20}$ or PGRP-LC ${ }^{E 12}$ flies, in the absence of infection. Both young $(2-4 \mathrm{~d})$ and old $(30 \mathrm{~d})$ flies were examined. Means \pm SD are shown. Means were analyzed by ANOVA and separated for significance according to Fisher's protected LSD at $P=0.05$. Means designated with the same letter are not significantly different. (B) RT-qPCR analysis shows that axenic flies express lower levels of Diptericin, upd3, Socs36E, and puckered compared with CR flies in the absence of infection. Expression of these genes increased in old CR flies, but not old axenic flies. Means \pm SD are shown. Values significantly different from the wild-type CR flies in a $t$-test at $P<0.001$ are denoted for axenic wild type versus CR wild type (\#), CR Rel ${ }^{E 20}$ versus CR wild type $(\approx)$, and old CR wild type versus young CR wild type $\left({ }^{\star}\right) .(C)$ Basal upd3 expression was not detected in axenic flies, as revealed by the GFP signal of upd3Gal4 UAS-GFP flies. (D1) The guts of old (30 d) CR, but not axenic, esgGal4 UAS-GFP flies display increased epithelium renewal all along the gut. (D2) Large rounded escargot-positive cells accumulate in the guts of old CR, but not axenic, wild-type flies.

\section{Disruption of gut epithelium renewal by an entomopathogenic bacterium}

P. entomophila is an entomopathogenic bacterium that, unlike Ecc15, is lethal to flies within 1-2 d of ingestion at high dose (Vodovar et al. 2005). We investigated the impact of P. entomophila on gut homeostasis and observed that, following ingestion, the length of the gut was reduced compared with uninfected flies (Fig. 6A). Closer examination of these guts revealed that enterocytes were missing along the gut or severely damaged, as evidenced by the loss of nuclear localization of DAPI signal (Fig. 6A, magnification). Use of esgGal4, UAS-GFP demonstrated that, in contrast to Ecc15, epithelium renewal was not detected following ingestion of $P$. entomophila. Instead, the number of ISCs was reduced, suggesting that infection with $P$. entomophila provoked a loss of ISCs along the gut, particularly in the anterior midgut (Fig. 6B). Since ingestion of $P$. entomophila still induces expression of both Socs36E and puckered in the gut (Fig. 6C), we conclude that this bacterium disrupts epithelium renewal despite proper activation of the required pathways. Furthermore, this impact on epithelium renewal appears to be linked to its lethality. Mutations in the genes encoding the GacA:GacS two-component system render P. entomophila fully avirulent, and ingestion of a gacA mutant has no significant effect on the immune response (Liehl et al. 2006). In a similar manner, gacA derivatives did not affect the level of epithelium renewal (Fig. 6B7). A mutation in aprA, a gene encoding a metalloprotease abundantly produced by $P$. entomophila, attenuates its pathogenicity (Liehl et al. 2006). Interestingly, ingestion of $P$. entomophila aprA induced a significant level of epithelium renewal without dramatically decreasing the number of ISCs (Fig. 6B8), thus supporting a correlation between pathogenicity and absence of epithelium renewal. To further test this hypothesis, we performed a dose response assay with the fully virulent $P$. entomophila strain. At concentrations of $\mathrm{OD}_{600}=5$ or lower, ingestion of $P$. entomophila increased gut epithelium renewal to a level similar to that observed with a high dose of Ecc15 (Fig. 6B; Supplemental Fig. S5A). Epithelium renewal was not observed at higher doses $\left(\mathrm{OD}_{600}>\right.$ 12.5), and loss of ISCs was apparent at concentrations higher than $\mathrm{OD}_{600}=50$ (Fig. 6B; Supplemental Fig. S5A). This graded effect of $P$. entomophila on epithelium renewal correlates to the severity of gut damage and the level of fly mortality (Supplemental Fig. S5B). Interestingly, epithelium renewal was also disrupted when flies ingested a high dose of paraquat or SDS (Supplemental Fig. S5A). Collectively, these results confirm that increased epithelium renewal is primarily a response to 
Figure 6. $P$. entomophila $(P e)$ disrupts infection-induced epithelium renewal. $(A)$ DAPI staining revealed that $P$. entomophila-infected guts are shorter than unchallenged guts $16 \mathrm{~h}$ after ingestion. The gut of $P$. entomophila-infected flies is composed of regions devoid of enterocytes and remaining enterocytes display abnormalities, as indicated by the loss of nuclear staining for DAPI. (B1-B8) Epithelium renewal was not observed in the gut of esgGal4, UAS-GFP flies infected with high doses of $P$. entomophila $\left(\mathrm{OD}_{600}>\right.$ $25, B 3, B 4)$. (B3) Lack of ISCs was observed when flies were fed a high concentration of $P$. entomophila $\left(\mathrm{OD}_{600}=100\right)$. $(B 5, B 6)$ In contrast, infection with lower doses of $P$. entomophila $\left(\mathrm{OD}_{600}<5\right)$ stimulated epithelium renewal. The avirulent $P$. entomophila mutant gacA induced neither epithelium renewal nor the loss of ISCs (B7), while the aprA derivative induced a slight increase of epithelium renewal (B8). Guts of esgGal4, UAS-GFP were examined $16 \mathrm{~h}$ after infection. $(C)$ Infection with $P$. entomophila induced both JAK-STAT (Socs36E, monitored $4 \mathrm{~h}$ postinfection) and JNK (puckered, monitored 30 min post-infection) pathways in the gut, as revealed by RT-qPCR.
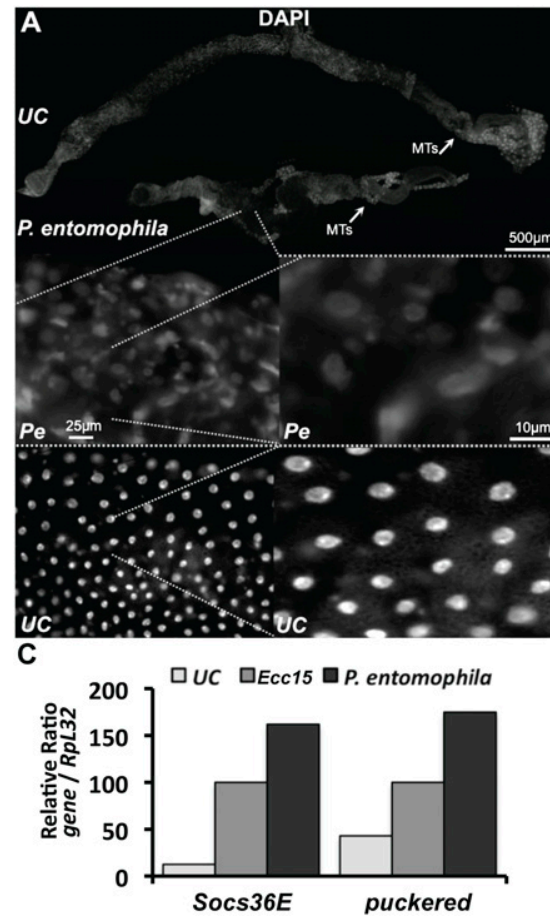

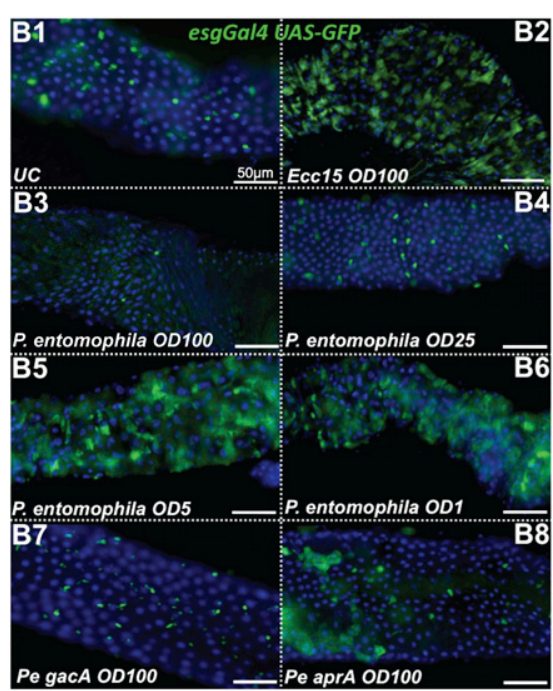

Pe gacA OD100 damage and that this response is inhibited above a certain threshold of damage.

\section{Discussion}

Our study demonstrates that both invasive and indigenous bacteria influence the level of epithelium turnover of the adult gut. Our results support a model that links the damage resulting from exposure to bacteria to ISC proliferation through JNK and JAK-STAT activation (see model in Fig. 7). The resulting increase in epithelium renewal is required to maintain gut homeostasis and survive bacterial infection, which demonstrates its essential role in host defense.

Previous studies have shown that the NADPH oxidase Duox plays an essential role in Drosophila gut immunity by generating microbicidal effectors such as ROS to eliminate both invasive and dietary microbes (Ha et al. 2005a, 2009). Ecc15 is a potent activator of Duox, which in turn is important in the clearance of this bacterium $(\mathrm{Ha}$ et al. 2005a). This oxidative burst is coordinated with the induction of many genes involved in ROS detoxification upon Ecc15 ingestion (Buchon et al. 2009). Here, we provide evidence that the observed increase in epithelium renewal upon Ecc15 infection is a compensatory mechanism that repairs the damage inflicted to the gut by this oxidative burst. This is supported by our observation that reducing ROS levels by either the ingestion of antioxidants or silencing the Duox gene reduces epithelium renewal. Although ISC proliferation could be directly triggered by ROS, it is more likely a consequence of signals produced by stressed enterocytes. A number of data support this hypothesis: (1) Ingestion of corrosive agents can also induce ISC proliferation (Biteau et al. 2008; Amcheslavsky et al. 2009; Buchon et al. 2009), and (2) physical injury is sufficient to induce local activation of the cytokine Upd3, which promotes epithelium renewal. Interestingly, a significant increase in epithelium renewal was observed in Duox RNAi flies at late time points following infection (Supplemental Fig. S1D), correlating with damage attributed to the proliferation of Ecc15 in the guts of Duox-deficient flies (Ha et al. 2005a). While the increase in epithelium renewal observed with Ecc15 is clearly linked to the damage induced by the host immune response, it is likely that effects on epithelium renewal by other pathogens could be more direct and mediated by virulence factors, such as the production of cytolytic toxins.

Our data indicate that the JAK-STAT and JNK pathways synergize to promote ISC proliferation and epithelium renewal in response to the damage induced by infection. The JAK-STAT pathway is implicated in the regulation of stem cells in multiple tissues and is proposed to be a common regulator of stem cell proliferation (Gregory et al. 2008). Our data extend this observation by showing that the JAK-STAT pathway is also involved in ISC activation upon bacterial infection. We demonstrate that the cytokine Upd3 is produced locally by damaged enterocytes and subsequently stimulates the JAK-STAT pathway in ISCs to promote their proliferation. Our results globally agree with a recent study showing that the JAK-STAT pathway is involved in ISC proliferation 


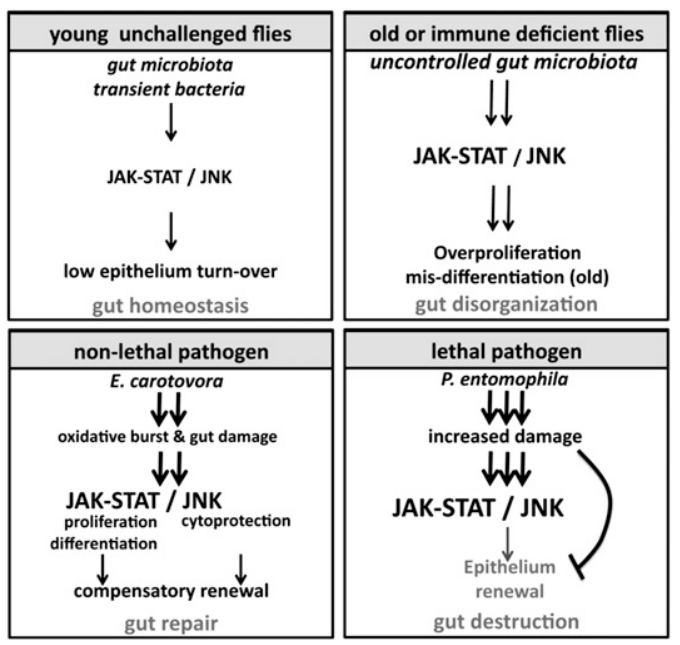

Figure 7. Modulation of gut epithelium renewal by invasive pathogens and indigenous gut microbiota. The JAK-STAT and JNK signaling pathways are required to maintain gut homeostasis upon exposure to a broad range of bacteria. In normal conditions, low levels of the indigenous gut microbiota and transient environmental microbes maintain a basal level of epithelium renewal. The increase in gut microbes in old or Imd-deficient flies is associated with a chronic activation of the JNK and JAK-STAT pathways, leading to an increase in ISC proliferation and gut disorganization. The impact of pathogenic bacteria can have different outcomes on gut homeostasis, depending on the degree of damage they inflict on the host. Damage to the gut caused by infection with E. carotovora is compensated for by an increase in epithelium renewal. Infection with a high dose of $P$. entomophila disrupts the homeostasis normally maintained by epithelium renewal and damage is not repaired, contributing to the death of the fly.

upon infection with a low dose of P. entomophila (Jiang et al. 2009). This work and our study clearly demonstrate that the JAK-STAT pathway adjusts the level of epithelium renewal to ensure proper tissue homeostasis by linking enterocyte damage to ISC proliferation. In the study by Jiang et al. (2009), they also uncover an additional role of this pathway in the differentiation of enteroblasts during basal gut epithelium turnover. The implication of the JAK-STAT pathway in differentiation could explain the accumulation of the small-nucleated escargot-positive cells we observed in the gut of flies with reduced JAK-STAT signaling in ISCs (Fig. 2C). The JAKSTAT pathway was also shown previously to control the expression of some antimicrobial peptides such as Drosomycin 3 (Dro3) (Buchon et al. 2009). Therefore, the JAK-STAT pathway has a dual role in the gut upon infection, controlling both the immune response and epithelium renewal.

Our data show that the lack of JNK pathway activity in ISCs results in the loss of ISCs in guts infected with Ecc15, thus preventing epithelium renewal. Our findings are consistent with the attributed function of JNK at the center of a signal transduction network that coordinates the induction of protective genes in response to oxidative challenge (Wang et al. 2003; Biteau et al. 2008). This cytoprotective role against ROS would protect ISCs from the oxidative burst induced upon Ecc15 infection, explaining why ISCs die by apoptosis when JNK activity is reduced. As suggested by Biteau et al. (2008), it is likely that JNK signaling is required not only to protect ISCs from oxidative stress, but also to induce stem cell proliferation to replace damaged differentiated cells. This is supported by the observation that overexpression of the JNKK Hep in ISCs is sufficient to trigger an epithelium renewal in the absence of infection. In addition, increased JNK activity in ISCs of old flies has been linked to hyperproliferative states and age-related deterioration of the intestinal epithelium (Biteau et al. 2008). Our study extends this observation by showing that JNK signaling is also required for epithelium renewal upon Ecc15 infection. Thus, infection with Ecc15 recapitulates in an accelerated time frame the impacts of increased stress observed in guts of aging flies

We observed that the inhibition of the dJun transcription factor in ISCs leads to a loss of stem cells in the absence of infection, suggesting that this transcription factor plays a critical role in ISC maintenance in the gut. We have no definitive explanation for why the dJun-IR construct behaves differently than the basket and hep-IR constructs. We speculate that this could be due to (1) differences in the basal activity of the JNK pathway, which would be blocked only with the $d J u n-I R$ that targets a terminal component of the pathway; (2) effects of Jun in ISCs independent of the JNK pathway, as reported in other systems (Bates et al. 2008); or (3) side effects of the dJun-IR construct.

In contrast to the requirement of the JNK pathway upon Ecc15 infection, Jiang et al. (2009) reported that oral ingestion with a low dose of $P$. entomophila still induced mitosis in the JNK-defective mutant hep ${ }^{1}$. In agreement, we found that inhibiting the JNK pathway in ISCs did not block the induction of epithelium renewal by a low dose of P. entomophila (Supplemental Fig. 3C). This difference in the requirement of the JNK pathway may be explained by the nature of these two pathogens. Whereas Ecc15 damages the gut through an oxidative burst that activates the JNK pathway, the stimulation of epithelium renewal by P. entomophila could be due to a more direct effect of this bacterium on the gut. Altogether, Jiang et al. (2009) and our study point to an essential role of the JAK-STAT pathway in modulation of epithelium renewal activity, while the role of JNK may be dependent on the infectious agent and any associated oxidative stress. While it is known that the JNK pathway is activated by a variety of environmental challenges including ROS, the precise mechanism of activation of this pathway has not been elucidated. Similarly, the molecular basis of upd3 induction in damaged enterocytes is not known. Future work should decipher the nature of the signals that activate these pathways in both ISCs and enterocytes, as well as the possible cross-talk between the JNK and JAK-STAT pathways in ISC control.

The observation that flies unable to renew their gut epithelium eventually succumb to Ecc15 infection highlights the importance of this process in the gut immune 
response. It is striking that defects in epithelium renewal are more detrimental to host survival than deficiency in the Imd pathway, even though this pathway controls most of the intestinal immune-regulated genes induced by Ecc15 (Buchon et al. 2009). Our results are in agreement with a previous study indicating that, in the Drosophila gastrointestinal tract, the Imd-dependent immune response is normally dispensable to most transient bacteria, but is provisionally crucial in the event that the host encounters ROS-resistant microbes (Ryu et al. 2006). However, our study demonstrates that efficient and rapid clearance of bacteria in the gut by Duox is possible only when coordinated with epithelium renewal to repair damage caused by ROS. This finely tuned balance between bacterial elimination by Duox activity and gut resistance to collateral damage induced by ROS is likely the reason why flies normally survive infection by Ecc15. Yet, this calibration also exposes a vulnerability that could easily be manipulated or subverted by other pathogens. Along this line, our work also exposes the range of impact different bacteria can have on stem cell activation. We observed that infection with high doses of $P$. entomophila led to a loss of gut integrity, including the loss of stem cells. Moreover, the ability of P. entomophila to disrupt epithelium renewal correlates with damage to the gut and the death of the host. Since both JNK and JAK-STAT pathways are activated upon infection with $P$. entomophila, this suggests that this bacterium activates the appropriate pathways necessary to repair the gut, but ISCs are unable to respond accordingly. Interestingly, a completely avirulent $P$. entomophila mutant (gacA) does not persist in the gut and does not induce epithelium renewal. In contrast, an attenuated mutant (aprA) somewhat restores epithelium renewal. These observations, along with the dose response analysis using $P$. entomophila and corrosive agents, suggest that the virulence factors of this entomopathogen disrupt epithelium renewal through excessive damage to the gut. Of note, recent studies suggest that both Helicobacter pylori and Shigella flexneri, two bacterial pathogens of the human digestive tract, interfere with epithelium renewal to exert their pathological effects (Iwai et al. 2007; Mimuro et al. 2007). This suggests that epithelium renewal could be a common target for bacteria that infect through the gut (Chatterjee and Ip 2009; Cronin et al. 2009; Jiang et al. 2009). In this respect, the host defense to oral bacterial infection could be considered as a bimodular response, composed of both immune and homeostatic processes that require strict coordination. Disruption of either process results in the failure to resolve the infection and impedes the return to homeostasis.

In contrast to the acute invasion by pathogenic bacteria, indigenous gut microbiota are in constant association with the gut epithelium, and thus may impact gut homeostasis. Using axenically raised flies, we established that indigenous microbiota stimulate a basal level of epithelium renewal that correlates with the level of activation of the JAK-STAT and JNK pathways. This raises the possibility that both indigenous and invasive bacteria, such as Ecc15, are capable of triggering epithe- lium renewal by the same process. Additionally, our data support a novel homeostatic mechanism in which the density of indigenous bacteria is coupled to the level of epithelium renewal. This is the first report that gut microbiota affect stem cell activation and epithelium renewal, concepts proposed previously in mammalian systems (Hooper 2004) but never fully demonstrated. This also implies that variations in the level of epithelium renewal observed in different laboratory contexts could actually be due to impacts from gut microbes.

Importantly, in this context, we show that lack of indigenous microbiota reverts most age-related deterioration of the gut. Aging of the gut is usually marked by both hyperproliferation of ISCs and differentiation defaults that lead to disorganization of the gut epithelium (Biteau et al. 2008; N Choi et al. 2008). These alterations have been shown to be associated with activation of the PDGF- and VEGF-related factor $2(\mathrm{Pvf} 2) / \mathrm{Pvr}$ and JNK signaling pathways directly in ISCs (Biteau et al. 2008; N Choi et al. 2008). Accordingly, inhibition of the JNK pathway in ISCs fully reverts the epithelium alterations that occur with aging (Biteau et al. 2008). This raises the possibility that gut microbiota could exert their effect through prolonged activation of the JNK pathway. Interestingly, immune-deficient flies, lacking the Imd pathway, also display hyperproliferative guts and have higher basal levels of activation of the JNK and JAK-STAT pathways. The observation that these flies also harbor higher numbers of indigenous bacteria further supports a model in which failure to control gut microbiota leads to an imbalance in gut epithelium turnover. Future work should analyze the mechanisms by which gut microbiota affect epithelium renewal and whether this is due to a direct impact of bacteria on the gut or is mediated indirectly through changes in fly physiology. Moreover, the correlation between higher numbers of indigenous bacteria and increased disorganization of the gut upon aging in flies lacking the Imd pathway raises the possibility that a main function of this pathway is to control gut microbiota. This is in agreement with concepts emerging in mammals that support an essential role of the gut immune response in maintaining the beneficial nature of the host-microbiota association (Hooper 2009). This function also parallels the theory of "controlled inflammation" described in mammals, where a low level of immune activation is proposed to maintain gut barrier integrity (Sansonetti 2004; Pedron and Sansonetti 2008).

In conclusion, this study unravels some of the complex interconnections between the immune response, invasive and indigenous microbiota, and stem cell homeostasis in the gut of Drosophila. Based on the evolutionary conservation of transduction pathways such as JNK and JAK-STAT between Drosophila and mammals, it is likely that similar processes occur in the gut of mammals during infection. Interestingly, stimulation of stem cell activity by invasive bacteria is proposed to favor the development of hyperproliferative states found in precancerous lesions (Macdonald and Monteleone 2005; Radtke and Clevers 2005). Thus, Drosophila may provide a more accessible model to elucidate host mechanisms to 
maintain homeostasis and the impact of bacteria on this process.

\section{Materials and methods}

Fly stocks and rearing

For description of the stock genotypes, specificities of the Gal4 drivers, and efficiency of RNAi lines used in this study, see the Supplemental Material. Drosophila stocks were maintained at $23^{\circ} \mathrm{C}$ using standard fly medium (maize flour, dead yeast, agar, and fruit juice) devoid of living yeast. RNAi constructs were validated by RT-qPCR (Supplemental Fig. S7). Axenic Canton, Oregon, esgGal4, UAS-GFP, Rel ${ }^{E 20}$, and PGRP-LC E12 stocks were generated by bleaching embryos and maintaining embryos and emerging flies on autoclaved fly medium. The presence of bacteria in gut homogenates was examined by PCR amplification of 16S rRNA genes using eubacterial primers $(27 \mathrm{~F}$ and $1492 \mathrm{R})$, and by culturing the homogenates on mannitol agar or 1/10-strength tryptic soy agar.

\section{Bacterial strains}

Ecc15 is a Gram-negative bacterium that induces a strong local immune response (Basset et al. 2000; Buchon et al. 2009). P. entomophila is a Gram-negative bacterium that is lethal to Drosophila when ingested (Vodovar et al. 2005; Liehl et al. 2006).

\section{Infection and compound feeding}

Bacterial infections were performed as described elsewhere (Buchon et al. 2009). For oral infection, flies were incubated $2 \mathrm{~h}$ at $29^{\circ} \mathrm{C}$ in an empty vial and then placed in a fly vial with food solution and maintained at $29^{\circ} \mathrm{C}$. The food solution was obtained by mixing a pellet of an overnight culture of bacteria $\left(\mathrm{OD}_{600}=\right.$ 200), $10 \mathrm{mM}$ paraquat (Promega), or $0.2 \% \mathrm{H}_{2} \mathrm{O}_{2}$ (Sigma) with a solution of $5 \%$ sucrose (1:1), added to a filter disk that completely covered the surface of standard fly medium. Flies were incubated for $2 \mathrm{~d}$ at $29^{\circ} \mathrm{C}$ on the contaminated filter, or 1 $\mathrm{d}$ for survival analysis, after which they were transferred to fresh vials. For antioxidant treatments, flies were fed a mixture of bacteria (final $\left.\mathrm{OD}_{600}=100\right)$ and sucrose $(2.5 \%$ final), to which glutathione (0.5 mM final; Sigma) or NAC (20 mM final; Sigma) was added; control flies were fed bacteria and sucrose or sucrose alone.

\section{Clonal analysis}

The marked lineage system developed by Harrison and Perrimon (1993) was used to generate clones of lacZ-expressing cells (Ohlstein and Spradling 2006). See the Supplemental Material for details. Adults of the genotype yw, hs-FLP; X-15-33/X-15-29 were produced by standard crosses. To induce somatic recombination, 3-d-old adult flies were heat-shocked for $60 \mathrm{~min}$ at $37^{\circ} \mathrm{C}$ on three consecutive days. A day after, flies were orally infected with Ecc15 or fed on sucrose. Guts were dissected $10 \mathrm{~d}$ after infection and stained with X-gal (Romeo and Lemaitre 2008).

\section{Live imaging and immunofluorescence}

For live imaging, guts were dissected at room temperature in $1 \times$ phosphate-buffered saline (PBS) and immediately mounted in the anti-fading agent AF1 (Citifluor). Samples were observed for fluorescence with an Axioplot imager Z1 and Axiocam mRM camera (Zeiss). For immunofluorescence, guts were dissected in
$1 \times$ PBS; fixed for $20 \mathrm{~min}$ in PBS, $0.1 \%$ Tween $20(\mathrm{PBT})$, and $4 \%$ paraformaldehyde; and then stained with primary antibody (antiPH3 [1/1000; Upstate Biotechnologies/Millipore], anti-cleaved Caspase-3 [1/500; Asp175, Cell signal], anti-GFP [1/1000; Roche Diagnostics], anti- $\beta$-galactosidase [1/500; Sigma], anti-Delta [1/ 5000], anti-Prospero [1/500] [Developmental Studies Hybridoma Bank]) in PBT + BSA. Secondary staining was performed with Alexa488 or Alexa594-coupled anti-mouse or anti-rabbit antibodies (Invitrogen). DNA was stained with DAPI (Sigma).

\section{RT-qPCR}

Total RNA was extracted from 40 dissected guts using Trizol (Invitrogen). RT-qPCR was performed using SYBR Green I (Roche) on a LightCycler 2.0 as described previously (Buchon et al. 2009). The amount of mRNA detected was normalized to control $R p L 32$ values. Primers used to monitor mRNA quantification can be obtained on request.

\section{Acknowledgments}

We thank our colleague J.P. Boquete for technical support; M. Meister (Strasbourg) for stimulating discussions and the gift of the UAS-upd3 lines; W.-J. Lee (Seoul) for comments on the manuscript; and R. Ueda (National Institute of Genetic, Mishima, Japan), M. Crozatier (Toulouse), A. Bardin (Paris), F. Leulier (Gifsur-Yvette), J.E. Darnell (New York), and C.A. Micchelli (St. Louis) for fly stocks. This work was funded by the ERC Advanced Grant and the Swiss National Fund (3100A0-12079/1).

\section{References}

Agaisse H, Petersen UM, Boutros M, Mathey-Prevot B, Perrimon N. 2003. Signaling role of hemocytes in Drosophila JAK/STATdependent response to septic injury. Dev Cell 5: 441-450.

Amcheslavsky A, Jiang J, Ip YT. 2009. Tissue damage-induced intestinal stem cell division in Drosophila. Cell Stem Cell 4: 49-61.

Bach EA, Ekas LA, Ayala-Camargo A, Flaherty MS, Lee H, Perrimon N, Baeg GH. 2007. GFP reporters detect the activation of the Drosophila JAK/STAT pathway in vivo. Gene Expr Patterns 7: 323-331.

Basset A, Khush RS, Braun A, Gardan L, Boccard F, Hoffmann JA, Lemaitre B. 2000. The phytopathogenic bacteria Erwinia carotovora infects Drosophila and activates an immune response. Proc Natl Acad Sci 97: 3376-3381.

Bates KL, Higley M, Letsou A. 2008. Raw mediates antagonism of AP-1 activity in Drosophila. Genetics 178: 1989-2002.

Biteau B, Hochmuth CE, Jasper H. 2008. JNK Activity in somatic stem cells causes loss of tissue homeostasis in the aging Drosophila gut. Cell Stem Cell 3: 442-455.

Brown S, Hu N, Hombria JC. 2001. Identification of the first invertebrate interleukin JAK/STAT receptor, the Drosophila gene domeless. Curr Biol 11: 1700-1705.

Buchon N, Broderick NA, Poidevin M, Pradervand S, Lemaitre B. 2009. Drosophila intestinal response to bacterial infection: Activation of host defense and stem cell proliferation. Cell Host Microbe 5: 200-211.

Chatterjee M, Ip YT. 2009. Pathogenic stimulation of intestinal stem cell response in Drosophila. I Cell Physiol 220: 664671.

Choi N, Kim J, Yang D, Kim Y, Yoo M. 2008. Age-related changes in Drosophila midgut are associated with PVF2, a PDGF/VEGF-like growth factor. Aging Cell 7: 318-334.

Choi YJ, Hwang MS, Park JS, Bae SK, Kim YS, Yoo MA. 2008. Age-related upregulation of Drosophila caudal gene via 
NF-кB in the adult posterior midgut. Biochim Biophys Acta 1780: $1093-1100$

Cronin SJ, Nehme NT, Limmer S, Liegeois S, Pospisilik JA, Schramek D, Leibbrandt A, Simoes RD, Gruber S, Puc U, et al. 2009. Genome-wide RNAi screen identifies genes involved in intestinal pathogenic bacterial infection. Science 325: 340-343.

Gregory L, Came PJ, Brown S. 2008. Stem cell regulation by JAK/STAT signaling in Drosophila. Semin Cell Dev Biol 19: 407-413.

Ha E, Oh C, Bae YS, Lee WJ. 2005a. A direct role for dual oxidase in Drosophila gut immunity. Science 310: 847-850.

Ha E, Oh C, Ryu J, Bae YS, Kang SW, Jang IH, Brey P, Lee WJ 2005b. An antioxidant system required for host protection against gut infection in Drosophila. Dev Cell 8: 125132.

Ha EM, Lee K, Park SH, Kim SH, Nam HJ, Lee HY, Kang D, Lee WJ. 2009. Regulation of DUOX by the Gaq-phospholipase $\mathrm{C} \beta-\mathrm{Ca}^{2+}$ pathway in Drosophila gut immunity. Dev Cell 16: 386-397.

Harrison DA, Perrimon N. 1993. Simple and efficient generation of marked clones in Drosophila. Curr Biol 3: 424-433.

Hooper LV. 2004. Bacterial contributions to mammalian gut development. Trends Microbiol 12: 129-134.

Hooper LV. 2009. Do symbiotic bacteria subvert host immunity? Nat Rev Microbiol 7: 367-374.

Iwai $\mathrm{H}$, Kim $M$, Yoshikawa $\mathrm{Y}$, Ashida $\mathrm{H}$, Ogawa $\mathrm{M}$, Fujita $\mathrm{Y}$, Muller D, Kirikae T, Jackson PK, Kotani S, et al. 2007. A bacterial effector targets Mad2L2, an APC inhibitor, to modulate host cell cycling. Cell 130: 611-623.

Jiang $\mathrm{H}$, Patel $\mathrm{PH}$, Kohlmaier A, Grenley MO, McEwen DG, Edgar BA. 2009. Cytokine/Jak/Stat signaling mediates regeneration and homeostasis in the Drosophila midgut. Cell 137: 1343-1355.

Krzemień J, Dubois L, Makki R, Meister M, Vincent A, Crozatier M. 2007. Control of blood cell homeostasis in Drosophila larvae by the posterior signaling centre. Nature 446: 325-328.

Lee T, Luo L. 2001. Mosaic analysis with a repressible cell marker (MARCM) for Drosophila neural development. Trends Neurosci 24: 251-254.

Liehl P, Blight M, Vodovar N, Boccard F, Lemaitre B. 2006. Prevalence of local immune response against oral infection in a Drosophila/Pseudomonas infection model. PLoS Pathog 2: e56. doi: 10.1371/journal. ppat.0020056.

Macdonald TT, Monteleone G. 2005. Immunity, inflammation, and allergy in the gut. Science 307: 1920-1925.

Martín-Blanco E, Gampel A, Ring J, Virdee K, Kirov N, Tolkovsky AM, Martinez-Arias A. 1998. puckered encodes a phosphatase that mediates a feedback loop regulating JNK activity during dorsal closure in Drosophila. Genes \& Dev 12: $557-$ 570.

Micchelli CA, Perrimon N. 2006. Evidence that stem cells reside in the adult Drosophila midgut epithelium. Nature 439: 475-479.

Mimuro H, Suzuki T, Nagai S, Rieder G, Suzuki M, Nagai T, Fujita Y, Nagamatsu K, Ishijima N, Koyasu S, et al. 2007. Helicobacter pylori dampens gut epithelial self-renewal by inhibiting apoptosis, a bacterial strategy to enhance colonization of the stomach. Cell Host Microbe 2: 250-263.

Ohlstein B, Spradling AC. 2006. The adult Drosophila posterior midgut is maintained by pluripotent stem cells. Nature 439: 470-474.

Park JS, Kim YS, Yoo MA. 2009. The role of p38b MAPK in agerelated modulation of intestinal stem cell proliferation and differentiation in Drosophila. Aging 1: 637-651.
Pedron T, Sansonetti PJ. 2008. Commensals, bacterial pathogens and intestinal inflammation: An intriguing ménage à trois. Cell Host Microbe 3: 344-347.

Radtke F, Clevers H. 2005. Self-renewal and cancer of the gut: Two sides of a coin. Science 307: 1904-1909.

Ren C, Webster P, Finkel SE, Tower J. 2007. Increased internal and external bacterial load during Drosophila aging without life-span trade-off. Cell Metab 6: 144-152.

Romeo Y, Lemaitre B. 2008. Drosophila immunity: Methods for monitoring the activity of toll and imd signaling pathways. Methods Mol Biol 415: 379-394.

Ryu J, Ha E, Oh C, Seol J, Brey P, Jin I, Lee D, Kim J, Lee D, Lee WJ. 2006. An essential complementary role of NF-кB pathway to microbicidal oxidants in Drosophila gut immunity. EMBO J 25: 3693-3701.

Sansonetti PJ. 2004. War and peace at mucosal surfaces. Nat Rev Immunol 4: 953-964.

Vodovar N, Vinals M, Liehl P, Basset A, Degrouard J, Spellman P, Boccard F, Lemaitre B. 2005. Drosophila host defense after oral infection by an entomopathogenic Pseudomonas species. Proc Natl Acad Sci 102: 11414-11419.

Wang MC, Bohmann D, Jasper H. 2003. JNK signaling confers tolerance to oxidative stress and extends lifespan in Drosophila. Dev Cell 5: 811-816.

Zaidman-Remy A, Herve M, Poidevin M, Pilifloury S, Kim MO, Blanot D, Oh BH, Ueda R, Mengin-Lecreulx D, Lemaitre B. 2006. The Drosophila amidase PGRP-LB modulates the immune response to bacterial infection. Immunity 24: 463-473. 


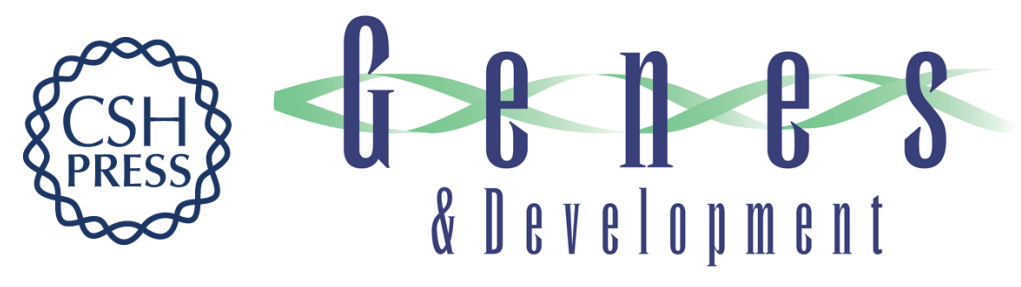

\section{Invasive and indigenous microbiota impact intestinal stem cell activity through multiple pathways in Drosophila}

Nicolas Buchon, Nichole A. Broderick, Sveta Chakrabarti, et al.

Genes Dev. 2009, 23:

Access the most recent version at doi:10.1101/gad.1827009

\section{Supplemental http://genesdev.cshlp.org/content/suppl/2009/10/02/23.19.2333.DC1 Material}

Related Content

Bacterial-modulated host immunity and stem cell activation for gut homeostasis Won-Jae Lee

Genes Dev. October , 2009 23: 2260-2265

References This article cites 39 articles, 8 of which can be accessed free at:

http://genesdev.cshlp.org/content/23/19/2333.full.html\#ref-list-1

Articles cited in:

http://genesdev.cshlp.org/content/23/19/2333.full.html\#related-urls

\section{License}

Email Alerting

Service

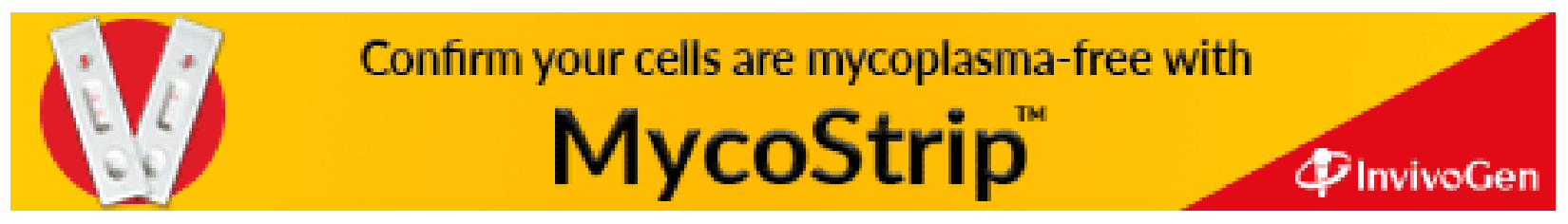

